\title{
Análisis de la relación entre la cultura organizacional y la planeación estratégica en el sector de la construcción en Bucaramanga y su área metropolitana
}

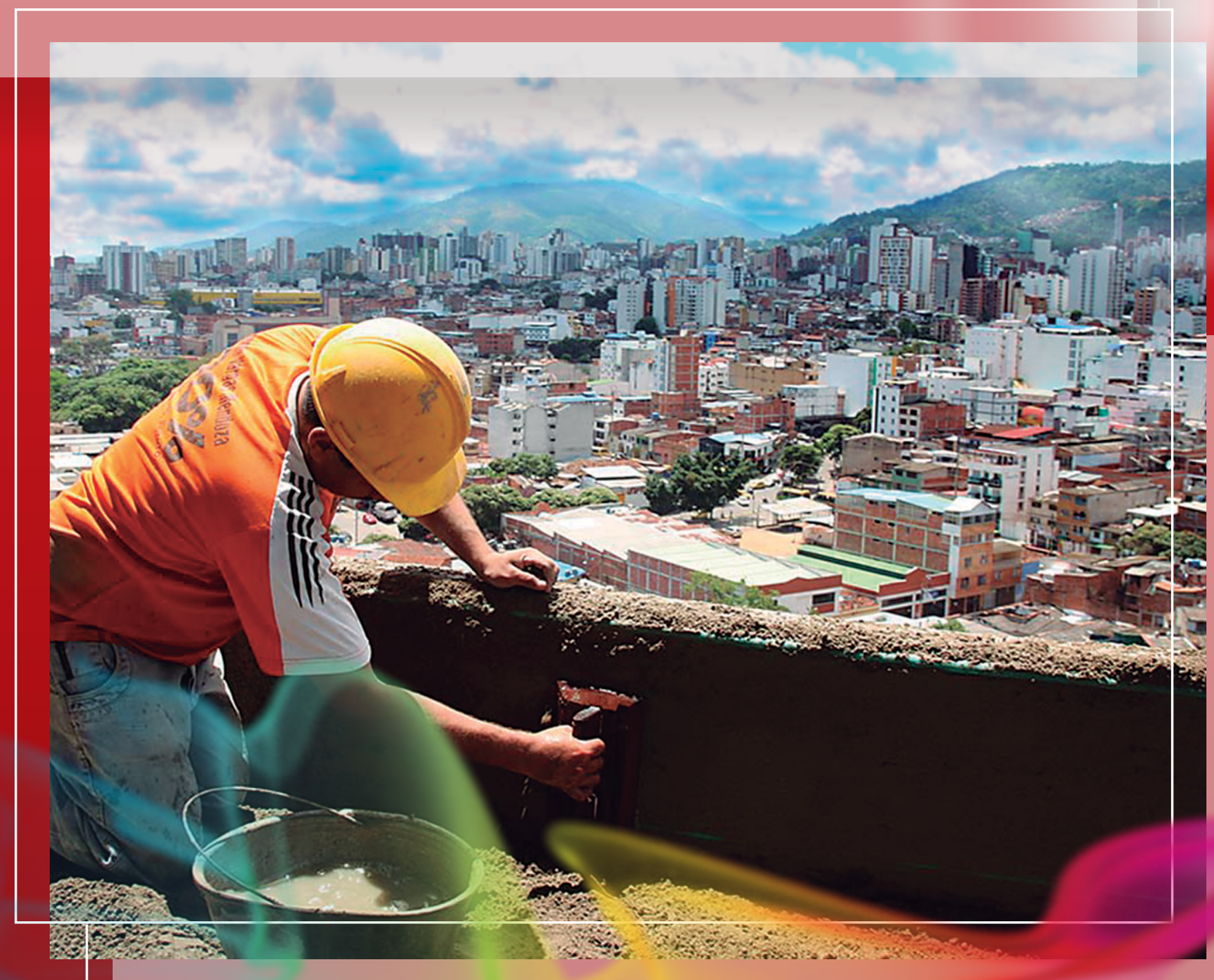




\title{
Análisis de la relación entre la cultura organizacional y la planeación estratégica en el sector de la construcción en Bucaramanga y su área metropolitana ${ }^{1}$
} Analysis of organizational culture and strategic planning relationship in building sector in Bucaramanga and its metropolitan area.

\author{
Lucelly Carolina Meza-Ariza ${ }^{2}$, José Gregorio Camargo Restrepo ${ }^{3 .}$ \\ Universitaria de Investigación y Desarrollo (UDI) Bucaramanga, Colombia.
}

Citación del artículo: Meza, L. \& Camargo, J. (2015). Análisis de la relación entre la cultura organizacional y la planeación estratégica en el sector de la construcción en Bucaramanga y su área metropolitana. I+D Revista de Investigaciones, 6(2), 100-110.

\section{Resumen}

El artículo nace de una consultoría realizada con el fin de analizar la cultura organizacional en empresas del sector de la construcción en la ciudad de Bucaramanga, a través de un sondeo, con el objetivo de determinar la alineación entre la planeación estratégica y la cultura de la organización. Para realizar el análisis, se inició la investigación en una de las empresas más representativas de la ciudad en el sector de la construcción, elegida por su trayectoria, calidad y buen nombre. Se tomó una muestra representativa de empleados directos y se analizó inicialmente a través del cuestionario de
Cameron \& Quinn (2011) según el modelo de los valores en competencia (Sepúlveda Laurence, 2004) esto con el fin de generar un diagnóstico de la cultura actual de la compañía. A continuación se aplicó un cuestionario basado en cinco dimensiones (organización y puesto de trabajo como componentes de la planeación estratégica y valores, motivación y comunicación como componentes de la C.O.) para determinar si la cultura presente está alineada a la planeación estratégica. Por último, se plantearon estrategias pertinentes para cada caso, con el fin de alinear las variables y encaminarlas hacia el aumento del desempeño organizacional como factor de diferenciación y competitividad.

\footnotetext{
${ }^{1}$ Artículo de investigación de enfoque cuantitativo, resultado de un proyecto de investigación culminado, perteneciente al área de Ciencias Sociales, desarrollado en el grupo de investigación Porter y financiado por la Universitaria de Investigación y Desarrollo (UDI) Bucaramanga (Colombia). Dirección: Calle 9 No. 23-55, PBX:6352525 ext. 104. Fecha de inicio: enero de 2015, fecha de terminación: junio de 2015.

${ }^{2}$ Administradora de Empresas, Universidad Santo Tomás. Estudiante de Maestría en Gestión Humana y Desarrollo Organizacional, Universidad Externado de Colombia. Docente del grupo Porter. Universitaria de Investigación y Desarrollo (UDI) Bucaramanga (Colombia). Dirección: Calle 9 No. 23-55 PBX: 6352525 ext. 104. Correo electrónico institucional:1meza1@udi.edu.co

${ }^{3}$ Ingeniero Industrial, Universidad Industrial de Santander. Estudiante de Maestría en Gerencia de Negocios, Universidad Industrial de Santander. Docente del grupo Porter. Universitaria de Investigación y Desarrollo (UDI), Bucaramanga (Colombia). Dirección: Calle9 No. 23-55 PBX:6352525 ext. 104. Correo electrónico institucional: jcamargo2@udi.edu.co
} 
Palabras clave: cultura organizacional, motivación, estructura organizacional, planeación estratégica, comunicación organizacional.

\section{Abstract}

This paper has been conceived from a consulting that has been intended to the analysis of organizational culture in companies in the building sector of Bucaramanga, Colombia performed through a survey and with the objective of determining the current alignment between strategic planning and the culture of the Organization in study. To perform a meaningful analysis this study analyzes a representative company of this city that is well known by its size and good will. A sample of employees was taken and analyzed initially through the questionnaire by Cameron and Quin (Cameron \& Quinn, 2011) under the competing values model to generate a diagnosis of the current culture of the company. Then a questionnaire was applied based on five dimensions (Organization and job as strategic planning and values, motivation and communication components, such as components of the O.C.) to determine if this culture is aligned to strategic planning. Finally we raised relevant strategies for each case in order to align variables and direct them in enhancing organizational performance as a factor of differentiation and competitiveness.

Key words: Organizational culture, motivation, organizational structure, strategic planning, organizational communication.

\section{Introducción}

La investigación tiene como fin identificar la cultura organizacional, la planeación estratégica definida por la empresa y la coherencia existente entre la cultura y la planeación realizada.

\section{Objetivos de la investigación}

- Identificar la cultura presente en la empresa.

- Identificar los parámetros más relevantes de la planeación estratégica.

- Contrastar la cultura con la planeación para determinar la alineación existente.

El sector construcción se destaca por el aporte que hace a la economía santandereana, haciéndose por ello atractivo para el estudio, además, la demografía del personal contratado para la ejecución de las labores hacen que este posea unas características especiales para su manejo efectivo y la alineación con la planeación estratégica de la organización.

Entre los aspectos relevantes del sector construcción (edificaciones y obras civiles) se pudo notar que el ingreso promedio de los trabajadores de este sector se encuentra un $40 \%$ por encima del salario mínimo mensual legal (smmlv) para el año 2013, por tanto, cerca del $68 \%$ de las personas ocupadas en este sector tienen un ingreso laboral superior smmlv, incluso, sobrepasando el ingreso promedio encontrado en la industria manufacturera, en el sector comercio, y en hoteles y restaurantes. También se destaca que las personas ocupadas en el sector de la construcción manifiestan contar con cerca de ocho años de escolaridad, 
coincidiendo con el dato encontrado a nivel nacional del total de ocupados (el cual fue 8.25 años), estas y otras variables demográficas permiten identificar de qué manera la cultura de la población de la región incide en la cultura organizacional (Porras, Burgos, \& Guio, 2014).

\section{Cultura organizacional}

La cultura de una institución incluye los valores, creencias y comportamientos que se consolidan y comparten durante la vida empresarial. El estilo de liderazgo en el ámbito de la alta gerencia, las normas los procedimientos y las características generales de los miembros de la empresa completan la combinación de elementos que forman la cultura de una compañía (Gómez H. S., 2010; Serrate-Alfonso et al., 2014), por consiguiente cada organización tiene su propia cultura, distinta de las demás, lo que le da su propia identidad.

"La política empresarial, está determinada al entorno cultural, sociopolítico y económico en los cuales se ponen en práctica. E1 comportamiento empresarial tiene su desarrollo dentro de un marco cultural en el cual se realizan las interacciones entre la cultura y la empresa" (Vargas, 2007).

Por ende, conocer la cultura que domina la organización permite comprender y determinar los comportamientos de las personas en esa compañía. Fukuyama (1996) concluye que "La cultura inhibe el crecimiento de grandes compañías en algunas sociedades, lo permite en otras y estimula el surgimiento de nuevas formas de empresa económica", como se puede evidenciar a través de los autores anteriores la cultura organizacional influye directamente sobre el comportamiento de las personas y su desempeño.

Figura 1. Dimensiones de la cultura

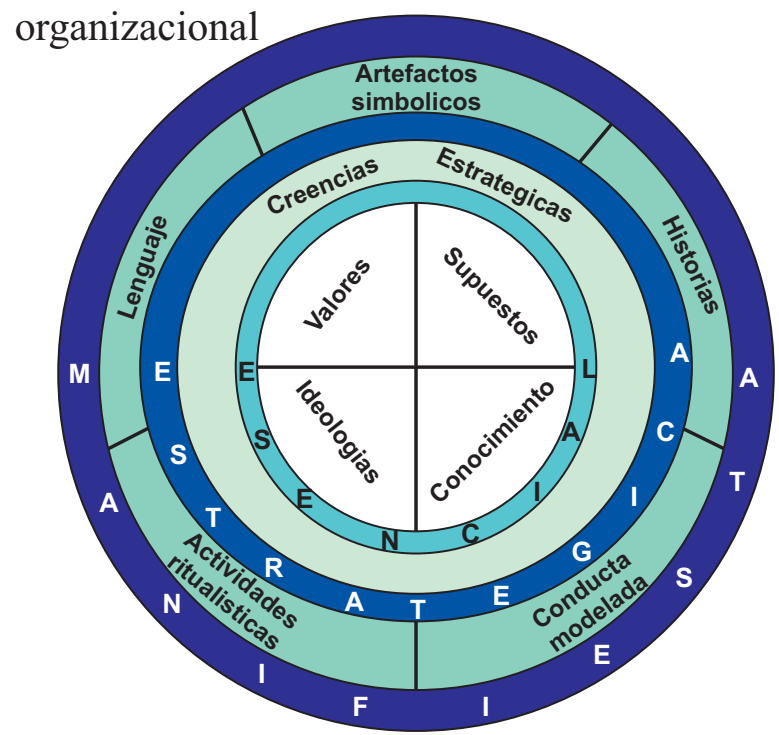

Fuente:(Toca \& Carrillo, 2009)

La cultura empresarial tiene tres niveles: los artefactos, los valores y los supuestos. Los artefactos son todo aquello que se puede ver, oír y sentir en un entorno cultural, como la arquitectura y el diseño, los símbolos, los rituales, y las historias, mitos y leyendas entre otros. Los valores enunciados y aprendidos son aquellos valores que la organización determina para el desarrollo de sus actividades, los cuales se han enunciado a través de la misión o visión de la empresa y que se desean transmitir en el comportamiento de los colaboradores, como el respeto y la amabilidad, entre otros. Por último están los supuestos de la cultura, que son aquellas conductas aprobadas o no por las personas que integran una organización pero que nunca han sido manifestadas por escrito; son conductas socialmente predispuestas para el comportamiento de las personas(Schein, 1988). 


\section{Planeación estratégica- P. E}

La planeación estratégica es el proceso de formular, implementar y evaluar decisiones multidisciplinarias que permiten que una empresa alcance sus objetivos, integrando la administración, el mercadeo, las finanzas y la contabilidad, la producción y las operaciones, la investigación y el desarrollo, y los sistemas de información para lograr alcanzar el éxito (Fred, 2013). El proceso de desarrollo de la P.E. se basa en la formulación de estrategias que implican desarrollar una visión y una misión, y establecer objetivos a largo plazo, que se han de seguir, además se complementan con diferentes tipos de planes que permiten guiar la organización (Fred, 2013).

\section{Alineamiento estratégico}

Es el proceso mediante el cual una organización construye una visión compartida y la hace realidad en la gestión diaria de la empresa.

Existen tres tipos de alineamiento estratégico (Gómez, 2010): Alineamiento vertical: es el conjunto de programas y acciones que una organización realiza para lograr que sus colaboradores conozcan e incorporen la estrategia a la empresa. Alineamiento horizontal: busca compatibilizar los procesos con las necesidades y expectativas de los clientes; para ello, las empresas definen la cadena del valor del negocio, identifican sus procesos clave y de soporte y los integran con las necesidades y expectativas del cliente. Alineamiento integral: integra la estrategia con los procesos, los colaboradores y los clientes. La estrategia se convierte en el centro y motor fundamental para lograr una visión compartida.

Teniendo en cuenta que el objetivo de la investigación es hacer un diagnóstico de sondeo del sector construcción que permita determinar si la planeación estratégica está o no alineada con la cultura organizacional, esta se basó en el alineamiento estratégico vertical.

\section{Metodología}

Con el fin de analizar las variables en las empresas se emplea el modelo 'Competing Values Framework' (Sepúlveda Laurence, 2004). Además, se implementó cuestionario 'Organizational Culture Assessment' de Cameron \& Quinn (2011), con el objetivo de realizar un diagnóstico inicial que permita identificar el tipo de cultura que posee la organización.

\section{Tipo de estudio}

La investigación es de carácter descriptivo, y su interés es el de describir las características de las empresas constructoras destacadas de la ciudad de Bucaramanga respecto a las variables cultura organizacional y planeación estratégica. Asimismo, es un estudio de enfoque cuantitativo, debido a que se recolectan datos para comprender el fenómeno.

\section{Participantes}

Una empresa constructora bastante representativa de la ciudad de Bucaramanga, sobresaliente y de tradición, dedicada a la construcción de bienes inmuebles (viviendas, edificios y afines).

Para la selección de la empresa se utilizó la base de datos de Camacol (Cámara Colombiana de la Construcción); valga aclarar que teniendo que la Cámara de Comercio no discrimina entre constructoras de vivienda, contratistas de obras 
civiles y constructoras de infraestructura, se decidió no hacer uso de su base de datos.

\section{Herramienta utilizada}

Se aplicaron dos cuestionarios tipo encuesta, el primero fue el Cuestionario de Cameron y Quinn Culture Assessment (OCAI) (Cameron \& Quinn, 2011), usado para determinar la cultura existente en la organización; el segundo cuestionario -basado en cuestionarios elaborados y validados anteriormente en otras investigaciones relacionadas, adaptados a las necesidades del objeto de estudio- es de elaboración propia, y su propósito es el de evaluar la alineación entre las variables planeación estratégica y cultura organizacional. Adicionalmente, se crearon preguntas específicas que permitieran medir las variables que conforman la cultura organizacional.

Algunas preguntas son tomadas de los siguientes cuestionarios y adaptadas según las necesidades de la investigación: cuestionario de clima laboral capacitación y desarrollo (Secretaría de Educación de Bolívar), encuesta de clima laboral y modelo de auditoría a la gestión humana (Manpower Group MeCA, 2012), y cuestionario

Cuestionario para la medición del componente de valores compartidos (Buendía Sierra, 2009).

El cuestionario se planteó en escala Likert de medición de actitudes. Esta es una escala fijada estructuralmente por dos extremos recorriendo un continuo, desde desfavorable hasta favorable con un punto medio neutral para cada afirmación (Casas Castañe, 1999).

Esta herramienta se considera una excelente opción para la medición de variables siguiendo el enfoque cuantitativo, particularmente para medir actitudes que permiten evaluar la posición del individuo y su comportamiento con el fin de determinar si existe o no alineación entre la planeación estratégica y a cultura organizacional. Para medir la percepción de los empleados frente a ellas y su alineación con la planeación estratégica de la organización, las variables se desglosaron en dimensiones; la clasificación se observa en la Tabla 1.

Tabla 1. Variables a evaluadas y sus dimensiones

\begin{tabular}{cccc}
\hline Planeación estratégica & Preguntas & Cultura organizacional & Preguntas \\
\hline Organización & $1-11$ & Valores & $12-15$ \\
Puesto de trabajo & $26-30$ & Motivación & $16-20$ \\
& & Comunicación & $21-25$ \\
\hline
\end{tabular}

Fuente: Los autores, según clasificación encuesta. 
Para calcular los resultados de las encuestas se promediaron las preguntas relacionadas con cada dimensión a fin de determinar su impacto positivo o negativo sobre la variable de estudio y su relación. El resumen del proceso se puede observar en la Tabla 2.

Tabla 2. Cálculo de los resultados arrojados por las encuestas

\begin{tabular}{cccl}
\hline Variables & Dimensiones & Preguntas & \\
\hline \multirow{2}{*}{ Planeación estratégica } & Organización & $1-11$ & Promedio D1 \\
& Puesto de trabajo & $26-30$ & Promedio D2 \\
Total variable 1 & Promedio & & \\
& Valores & $12-15$ & Promedio D3 \\
Cultura organizacional & Motivación & $16-20$ & Promedio D4 \\
& Comunicación & $21-25$ & Promedio D5 \\
Total variable 2 & Promedio & & \\
\hline
\end{tabular}

Fuente: Los autores.

\section{Tamaño muestral}

La organización objeto de estudio cuenta con doscientos empleados directos, se realizó un muestreo probabilístico con un nivel de confianza de $90 \%$ y un error del $5 \%$, dando como resultado 115 personas para la muestra, distribuidas de la siguiente manera:

Personal administrativo: 50 personas

Personal obras: 65 personas

\section{Procedimiento}

Se aplicó la herramienta de recolección de datos en las instalaciones de la empresa objeto de estudio con el fin de no modificar la percepción por cambios en el entorno de los empleados frente a la organización. La muestra del personal administrativo se llevó a cabo a través del muestreo en todos los departamentos ubicados en las instalaciones administrativas de la organización, entre los cuales se encuentran las áreas de recursos humanos, financiera, servicios generales, soporte legal, atención al cliente de IPQR calidad, y desarrollo urbano.
Para la obtención de la muestra del personal de obra se aplicaron las herramientas de recolección de datos a un grupo representativo de cuatro obras ubicadas en diferentes puntos de la ciudad de Bucaramanga.

\section{Resultados}

Los resultados arrojados por la herramienta de Cameron y Quinn (Organizational Culture Assessment) aplicada al personal administrativo de la empresa, permiten apreciar el estilo jerárquico como cultura dominante, lo que lleva a la organización a ser burocrática (alta estandarización y poca flexibilidad y muy eficiente, pero presentando resistencia al cambio, precisamente por el tipo de cultura. Este factor podría generar inconvenientes organizacionales en procesos de adaptación de la empresa a nuevas condiciones del entorno, además inhibe los procesos de innovación, que son uno de los principales factores diferenciadores en el sector.

Mediante la misma herramienta también se 
concluye que el personal de obras de la organización presenta características y rasgos del enfoque de clan, lo que facilita la coordinación de las diferentes actividades gracias al ajuste mutuo, a los comités de vinculación de áreas y a la interacción y comunicación tanto vertical como horizontal, lo cual, en conjunto, genera un ambiente de cooperativismo entre el personal. Pese a ello, la empresa debe tener control de las medidas implementadas en la organización para desarrollar el personal tanto en lo profesional como en lo personal, teniendo en cuenta que se puede volver paternalista, provocando que la compañía invierta en programas que no impactan en el desempeño y productividad de los empleados.

Otro factor que impacta a la organización de estudio es la jerarquía llevada en un alto grado de burocracia determinada por la estandarización de productos y servicios a través de un ambiente relativamente estable, con funciones integradas y coordinadas, con líneas claras en la toma de decisiones, basadas en el control y los mecanismos de responsabilidad, sin embargo, la burocracia puede ser una barrera para el desarrollo debido al entorno dinámico en el que se sumergen las empresas incentivado por adelantos tecnológicos, la globalización, las telecomunicaciones entre otros que modifican las condiciones del mercado provocando cambios y reingeniería en las empresas decididas a adaptarse y superar las dificultades existentes.

En la actualidad la empresa cuenta con una cultura organizacional burocrática, caracterizada por hacer un trabajo estructurado y formalizado con múltiples niveles jerárquicos, donde los empleados toman decisiones basados en los procedimientos con el fin de mantener la eficiencia, la fiabilidad y la producción constante. Además los trabajadores presentan una alta coordinación y un control de tiempos estricto siendo generalmente inflexibles.

La prueba desarrollada para la investigación permitió evaluar algunos factores de las necesidades del ser humano descritos por Maslow (1991); a continuación se presentan los factores de la pirámide de Maslow tenidos en cuenta: a través de la pregunta del salario se evalúa el cubrimiento de las necesidades fisiológicas descritas por; por medio de la pregunta de escala salarial se evalúa el factor de seguridad (económica); a través de la pregunta de las influencias y el comportamiento de los jefes se evalúa el factor de reconocimiento. El promedio obtenido en las preguntas permiten concluir que existe una brecha entre la satisfacción total y estado actual del empleado, lo que genera una disminución en la motivación y puede afectar el rendimiento, la productividad y el desempeño laboral.

Adicionalmente, en la pregunta sobre el efecto de las influencias en las promociones se evidencia e, se obtuvo una respuesta positiva, aspecto que permite identificar que la mayoría del personal de oficina percibe que la promoción de cargos está vinculada directamente con las relaciones interpersonales con directivos de la organización, factor que genera desmotivación y disminución del desempeño laboral, determinado por la creencia de que las habilidades y competencias no son suficientes para garantizar el ascenso dentro de la organización. 
Con respecto a la motivación, la prueba arroja puntajes ubicados en la media, debido a insatisfacciones en el personal de la empresa, exceptuando la primera pregunta, donde la mayoría del personal no considera que su trabajo esté bien remunerado, por lo cual el puntaje obtenido está por debajo de la media (3); este tipo de insatisfacción puede provocar fuga de personal capacitado a otras empresas del sector, al identificar mejores oportunidades en el mercado laboral. En estrecha relación con la pregunta anterior, se puede observar que el salario de los trabajadores no satisface las necesidades básicas de todos los empleados, debido al puntaje obtenido, situado a un punto por encima de la media $(3,1)$; esta situación podría ocasionar que los empleados busquen nuevas oportunidades laborales.

Otro aspecto relevante analizado es la necesidad de reconocimiento de la pirámide de Maslow, este aspecto está relacionado a las necesidades del ego o la autoestima, se afirma que todas las personas necesitan sentirse parte de un todo y ser apreciadas por el mismo, destacar y tener prestigio dentro del grupo social al cual pertenece (organización). Teniendo en cuenta lo anterior se plantearon dos preguntas para evaluar este aspecto fundamental en el desarrollo del empleado, la primer pregunta determina si el jefe tiene la capacidad de reconocer el buen desempeño de los trabajadores que tiene a cargo, donde se identifica a través del promedio que está ubicado cinco puntos por encima de la media $(3,5)$ el resultado refleja que algunos jefes realizan ese tipo de incentivo en su grupo de trabajo, sin embargo debería capacitarse al personal que cumple funciones de liderazgo de grupos a fin de satisfacer la necesidad presente.
La otra pregunta relacionada con tema es el proceso establecido para la promoción de cargos donde la mayoría de personas perciben que las influencias facilitan el ascenso dentro de la empresa, generando frustración al identificar este tipo de situaciones que impiden que se aprecien sus capacidades, también se evidencio a través de la herramienta de recolección de datos que algunos empleados en ocasiones disminuyen su productividad, este comportamiento afecta el desempeño organizacional.

\section{Conclusiones}

Los factores analizados en esta investigación influyen directamente en la composición del tipo de cultura organizacional, fomentando creencias y mitos en ocasiones equivocados, basados, en su mayoría, en la percepción de los líderes, por tanto se recomienda contrastar los valores corporativos y la planeación estratégica organizacional con la apreciación de los empleados para establecer las brechas existentes y diseñar estrategias que vayan alineando sus creencias con la visión de la empresa.

Además la insatisfacción de las necesidades de los empleados afecta el desempeño laboral, ejecutando actividades al mínimo esfuerzo derivado de la creencia sobre la falta de aprecio que tiene la organización de las habilidades, capacidades y competencias de los empleados que la integran por tanto se debería realizar análisis de clima organizacional y satisfacción laboral para identificar factores a mejorar e implementar acciones correctivas.

En general, la cultura organizacional del personal de la empresa objeto de estudio está 
alineada con la planeación estratégica, basada en que el personal conoce los parámetros organizacionales y se identifica con ellos; no obstante los resultados obtenidos en el estudio evidencian algunos desvíos entre la cultura organizacional y las directrices corporativas.

Además, los resultados muestran unos niveles de motivación medios, que se pueden mejorar a fin de impedir el detrimento de este factor, relevante e impulsor del desempeño laboral. Las recomendaciones anteriores proporcionan un parámetro para implementar estrategias que mejoren las condiciones laborales de los individuos pertenecientes a la organización, ayudando a aumentar la satisfacción laboral; sin embargo se sugiere hacer un control anual de la satisfacción laboral de los trabajadores a través del análisis de cultura y clima organizacional y la implementación de indicadores que permitan identificar posibles falencias.

\section{Referencias}

Blanco de Lossada, N. (2000). Instrumentos de recolección de datos primarios. Entrevistas, cuestionarios y Escala Lickert. En N.

Buendía Sierra, J. A. (Noviembre de 2009). Análisis organizacional de la dinámica interna de las empresas constructoras destacadas del Eje Cafetero. Manizales, Colombia.

Cameron, K., \& Quinn, R. (2011). Diagnosing and Changing Organizational Culture: Based on the Competing Values Framework. Estados Unidos: Third edition.
Casas Castañe, M. (15 de Julio de 1999). Cambios de actitudes en contextos interculturales. Modificación de prejuicios. Barcelona, España.

Fred, D. (2013). Conceptos de Administración Estratégica. México, México: Pearson Educación.

Gómez, H. S. (2010). La Gerencia Estratégica - teoría- Metodología- alineamiento, implementación y mapas estratégicos- Índices de gestión. 3R Editores.

Gómez, H. S. (2010). La gerencia estratégica. Teoría- metodología- alineamiento, implementación y mapas estratégicos. Índices de gestión. Bogotá, Colombia: 3R Editores.

Manpower Group MeCA. (2012). Guía de aplicación para las empresas. México.

Maslow, A. H. (1991). Motivación y personalidad. Madrid - España: Díaz de Santos.

Porras, M. P., Burgos, K. O., \& Guio, D. R. (2014). Características del mercado laboral en el sector de la construcción. Bogotá: Estudios Económicos Camacol.

Schein, E. (1988). La cultura empresarial y el liderazgo. Una visión dinámica. Barcelona, España: Plaza \& Janes.

Secretaría de Educación de Bolívar. (s.f.). Encuesta de clima laboral, capacitación y desarrollo. Recuperado el 02 de Marzo de 2015, de Encuesta Tick - Versión Gratuita:

http://www.portaldeencuestas.com/encuesta.ph 
$p ? i e=15784 \& i c=7015 \& c=1 d 489$

Sepúlveda Laurence, F. (Diciembre de 2004). El modelo competing values framework (CVF) y el diagnóstico de la cultura organizacional. Reingeniería de Procesos en Universidades. Recuperado el 05 de Marzo de 2015, de Universidad de Concepción: http://www2.udec.cl/ rea/REVISTA\%20PDF/R ev63/art1rea63.pdf

Serrate-Alfonso, A., PortuondoVélez, Á., SánchezPuigbert, N., \& Suárez-Ojeda, R. (2014). Evaluación de la cultura organizacional y su incidencia en la efectividad grupal. Ingeniería Industrial - Instituto Superior Politécnico José
Antonio, 2 - 12.

Toca, C. E., \& Carrillo, J. (2009). Asuntos teóricos y metodológicos de la cultura organizacional. Civilizar -Ciencias Sociales y Humanas, 121-130.

Vargas, J. (2007). La culturocracia organizacional en México. Jalisco, México: Edición Electrónica Gratuita. 